\title{
Associations between sleep duration, sleep quality and high sensitivity C-reactive protein among medical students of a medical college in Chhattisgarh, India
}

\author{
Gupta P. ${ }^{1}$, Choudhury A.B. ${ }^{2}$, Pawar S.M. ${ }^{3}$, Gopi K. ${ }^{4}$ \\ ${ }^{1}$ Pragati Gupta, second year M.B.B.S. student, ${ }^{2}$ Dr. Anil Baran Choudhury, Associate Professor, Department of \\ Biochemistry, ${ }^{3}$ Dr. Shankar Manohar Pawar, Professor, Department of Biochemistry, ${ }^{4}$ Keerti Gopi, Tutor, Department of \\ Biochemistry; all authors are affiliated with Government Medical College, Rajnandgaon (C.G.), India.
}

Corresponding Author: Dr. Anil Baran Choudhury, Associate Professor, Department of Biochemistry, Government Medical College, Rajnandgaon (C.G.). Email: ani.rai010@gmail.com

\begin{abstract}
Background: Recent studies in human have indicated possible pathophysiologic mechanisms to support the biologic plausibility of an association between sleep deprivation and future cardiovascular risk. In addition, emerging evidence suggests that inflammatory markers are elevated in sleep-deprived individuals. However, the relationship between sleep duration and inflammatory markers is still unclear. The aim of this study was to investigate associations between selfreported sleep duration, sleep quality, and high sensitivity C-reactive protein (hs-CRP) levels among medical students of a medical college in Chhattisgarh, India. Materials and Methods: A total of 150 Students (94 male, 56 female) of Government Medical College, Rajnandgaon, Chhattisgarh in the age group of 20 to 30 years were randomly enrolled in the present study. Blood samples were analyzed for fasting blood glucose and hs-CRP. Sleep duration and sleep quality was assessed using the Pittsburgh Sleep Quality Index (PSQI). Results: In the present study, a significant variation in serum hs-CRP levels with different sleep categories was observed $(\mathrm{P}<0.0001)$, with the hs-CRP levels being highest (Mean $\pm \mathrm{SD} ; 1.13 \pm 0.85 \mathrm{mg} / \mathrm{L}$ ) in short duration ( $<6$ hours) followed by long duration ( $>$ 8hours) sleepers (Mean $\pm \mathrm{SD}$; $0.79 \pm 0.41 \mathrm{mg} / \mathrm{L}$ ). In both linear and logistic regression analysis model adjusted for various risk factors for cardiovascular disease, short sleep duration was significantly associated with elevated serum hs-CRP levels. Conclusion: Our findings suggest that inflammatory mechanisms may play a role in the cardiovascular risk associated with sleep deprivation.
\end{abstract}

Keywords: Inflammatory markers, Sleep duration, High sensitivity C-reactive protein, Pittsburgh Sleep Quality Index, Cardiovascular disease

\section{Introduction}

Sleep is increasingly recognized as an important lifestyle contributor to health.The recommended hours of sleep for adults is 7 or more per night on a regular basis in order to maintain and improve health [1]. Chronic sleep debt, which is increasingly common in developed and developing countries, is associated with metabolic and endocrine alterations that may have pathological consequences in the long term [2].

Chronically insufficient sleep is endemic and has been declared as a public health problem by the Centres for Disease Control[3]. Medical students are one subgroup of the general population who appear to be especially

Manuscript received: $4^{\text {th }}$ November 2018

Reviewed: $14^{\text {th }}$ November 2018

Author Corrected: $20^{\text {th }}$ November 2018

Accepted for Publication: $23^{\text {rd }}$ November 2018 vulnerable to poor sleep, may be attributed to the long duration and high intensity of study, lack of proper knowledge of sleep, and high academic demands [4]. A number of studies in humans have indicated possible pathophysiologic mechanisms to support the biologic plausibility of an association between sleep deprivation and future cardiovascular risk $[5,6]$.

Serum high-sensitivity C-reactive protein (hs-CRP), a member of the pentraxin family of proteins and a nonspecific marker of low-grade inflammation [7], has been shown to independently predict cardiovascular disease [8]. CRP has been shown to enhance secretion of inflammatory mediators by vascular endothelium [9] and promote uptake of low-density lipoproteins through the induction and enhanced expression of macrophages 


\section{Original Research Article}

in atherosclerotic lesions [10]. The fact that inflammatory markers are elevated in sleep-deprived individuals [5,11-14], and treatment of sleep disorders reduces levels of inflammatory markers [15], suggesting that inflammatory mechanisms may play a role in the cardiovascular risk associated with sleep deprivation.

However, it is not clear whether this pro-inflammatory effect is observed with short-term sleep deprivation or persists chronically. To our knowledge, previous studies examining the associations between sleep duration, sleep quality and inflammatory markers were done either in general population or in non-medical students group and none of these studies were from Indian population.

Therefore, we performed a cross sectional study to investigate the associations between self-reported sleep duration, sleep quality, and serum level of hs-CRP among medical students of a medical college in Chhattisgarh, India. We also investigated whether the associations of sleep duration and sleep quality with hsCRP was independent of other risk factor for cardiovascular disease among these students.

\section{Materials \& Methods}

This study was conducted in the Department of Biochemistry, Government Medical College, Rajnandgaon, Chhattisgarh and approved by the Institutional Ethics Committee. 150 Students (94 male, 56 female) of Government Medical College, Rajnandgaon, Chhattisgarh in the age group of 20 to 30 years were recruited randomly for this study. Brief clinical history of present and past illness and medical therapy were recorded from all participants. Written informed consent was obtained from all the participants before entry into the study. The exclusion criteria were:

1. Those students were not willing to participate,

2. Self-reported depression, anxiety, or other psychiatric disorders or taking any medication related to these disorders,

3. History of obstructive sleep apnoea,

4. History of or suffering from any systemic illness or any infectious disease.

All the subjects were given a structured questionnaire, which included demographic information, diet, medical history, medication history, sleep assessment, and exercise habits. Sleep duration and sleep quality was assessed using the Pittsburgh Sleep Quality Index
(PSQI) [16]. A higher global PSQI score indicates poorer sleep quality, and a global PSQI score greater than five differentiates poor from good sleepers. Body mass index (BMI) was calculated for all subjects by using the formula weight in kilograms divided by the square of heights in metres.

Waist circumference (WC) and hip circumference were measured in the standing position using standard techniques and waist to hip ratio (WHR) was calculated as waist circumference divided by hip circumference. Systolic (SBP) and diastolic blood pressure (DBP) and heart rate were measured using an arm type fully automatic blood pressure monitoring system (Easy Care, Ravechi GmbH, Germany). Blood pressure was recorded in the sitting position in the right arm.

Venous blood samples were collected after an overnight fasting in the morning in an aseptic condition from antecubital vein. Blood samples were centrifuged at $-4^{\circ}$ centigrade and stored immediately at $-80^{\circ}$ centigrade until they were analysed. Fasting blood glucose (FBG) was estimated by the standard laboratory kit method (GOD-POD) using a fully automated biochemistry analyser (Beckman Coulter fully automated biochemistry analyzer AU680). Serum hs-CRP was measured by commercially available kit (Immunoturbidimetric method) using semi-automated analyzer (RMS analytica).

Statistical analysis: The Kolmogorov-Smirnov test was used to test the normality of the distribution. Variables with a skewed distribution were logtransformed before performing statistical analyses. Data were expressed as the mean \pm standard deviation. Comparison of baseline anthropometric and biochemical parameters across sleep duration subgroups were done using one-way ANOVA.Linear regression analysis was done to study association between self-reported sleep duration, sleep quality assessed by Pittsburgh sleep quality index (PSQI) and serum hs-CRP levels.

Multivariate logistic regression analysis was performed to evaluate association of both the sleep duration and sleep quality with hs-CRP risk groups. Additional regression models were adjusted for relevant covariates such as age, gender, BMI, waist circumference, waist to hip ratio, heart rate, SBP, DBP, and fasting blood glucose. All tests were two-tailed and $\mathrm{p}$ value less than 0.05 were considered to be statistically significant. All data were analyzed using statistical software SPSS version 19 (SPSS Inc., Chicago, IL, USA). 


\section{Results}

The baseline anthropometric and biochemical characteristics of study participants among different sleep duration subgroups are presented in Table-1. In all the study participants, a significant variation in hs-CRP with sleep categories was observed ( $p<0.0001)$, with the hs-CRP levels being highest (Mean \pm SD; $1.13 \pm 0.85 \mathrm{mg} / \mathrm{L}$ ) in short duration ( $<6$ hours) followed by long duration (>8hours) sleepers (Mean \pm SD; $0.79 \pm 0.41 \mathrm{mg} / \mathrm{L}$ ) (Table-1). No differences in BMI,waist circumference, waist to hip ratio, systolic blood pressure (SBP), diastolic blood pressure (DBP), heart rate, and fasting blood glucose (FBG) were observed.A total of 62 students, which represented 41.4 percent of total study participants, reported to have different levels of poor sleep quality as assessed by Pittsburgh sleep quality index (Table-2). In linear regression analysis model, short sleep duration was significantly associated with elevated serum hs-CRP levels which were true even after additional adjustment for other risk factors for cardiovascular disease (Table-3). Multivariate logistic regression analyses showed an significant inverse association of sleep duration with presence of elevated serum hs-CRP levels (more than $1 \mathrm{mg} / \mathrm{L}$ ) in age, gender and BMI-adjusted as well as age, gender BMI, WC, WHR, blood pressure, heart rate, fasting blood glucose adjusted model (odds ratio, adjusted for age, genderand BMI $=0.427$ (95\% confidence interval (CI), 0.261-0.699; $p=0.0007$; odds ratio, adjusted for age, BMI, WC, and WHR blood pressure, heart rate, fasting blood glucose, $=0.435$ (95\% CI, 0.263-0.718); $p=0.0011$ (Table-4). On the other hand, in both linear and logistic regression models, adjusted for all cardiovascular risk factors, poor sleep quality assessed by PSQI score was associated with elevated serum hs-CRP levels but associations were insignificant (Table-3 and Table-4).

Table-1: Baseline anthropometric and biochemical characteristics of study participants among different sleep duration sub-groups.

\begin{tabular}{|c|c|c|c|c|}
\hline \multicolumn{5}{|c|}{ Sleep duration group } \\
\hline & $<\mathbf{6}$ hours & 7hours & $>\mathbf{8 h o u r s}$ & $\boldsymbol{P}$ \\
\hline No. of participant(Male/Female) & $57(41 / 16)$ & $42(22 / 20)$ & $51(31 / 20)$ & \\
\hline Age (Years) & $21.32 \pm 1.97$ & $20.88 \pm 2.06$ & $20.80 \pm 1.81$ & NS \\
\hline BMI(Kg/m $\left.{ }^{2}\right)$ & $22.01 \pm 3.91$ & $21.65 \pm 3.08$ & $21.95 \pm 3.91$ & NS \\
\hline Waist Circumference(cm) & $82.32 \pm 9.42$ & $81.42 \pm 7.07$ & $82.45 \pm 10.01$ & NS \\
\hline Waist:hip ratio & $0.87 \pm 0.05$ & $0.87 \pm 0.05$ & $0.88 \pm 0.05$ & NS \\
\hline SBP $(\mathrm{mmHg})$ & $123.16 \pm 13.77$ & $121.43 \pm 16.32$ & $118.63 \pm 13.27$ & NS \\
\hline DBP $(\mathrm{mmHg})$ & $82.11 \pm 8.61$ & $78.10 \pm 11.31$ & $79.02 \pm 9.0$ & NS \\
\hline Heart rate(bpm) & $77.49 \pm 7.18$ & $78.57 \pm 5.92$ & $77.02 \pm 7.62$ & NS \\
\hline FBG $(\mathrm{mg} / \mathrm{dl})$ & $81.12 \pm 9.41$ & $81.77 \pm 11.01$ & $80.35 \pm 10.70$ & NS \\
\hline hs-CRP $(\mathrm{mg} / \mathrm{L})$ & $1.13 \pm 0.85$ & $0.74 \pm 0.29$ & $0.79 \pm 0.41$ & $<\mathbf{0 . 0 0 0 1}$ \\
\hline
\end{tabular}

Data are presented as the mean \pm SD (standard deviation). $p<0.05$ is significant; NS, not significant; SBP, systolic blood pressure; DBP, diastolic blood pressure; FBG, fasting blood glucose; hs-CRP, high sensitivity C-reactive protein.

Table-2: Frequency of sleep disturbance assessed by Pittsburgh sleep quality index score among study participant.

\begin{tabular}{|c|c|c|}
\hline PSQI score & n & Percent (\%) \\
\hline 5 & 32 & 21.3 \\
\hline 6 & 11 & 7.3 \\
\hline 7 & 5 & 3.3 \\
\hline 8 & 7 & 4.7 \\
\hline 9 & 4 & 2.7 \\
\hline 10 & 1 & 0.7 \\
\hline 11 & 1 & 0.7 \\
\hline 13 & 1 & 0.7 \\
\hline
\end{tabular}

PSQI, Pittsburgh sleep quality index; n, number of participants

*A total score of 5 or greater is indicative of poor sleep quality. 
Original Research Article

Table-3: Linear regression analysis for association of self reported sleep duration and sleep quality (PSQI score) with serum hs-CRP levels among study participants.

\begin{tabular}{|c|c|c|c|c|}
\hline & \multicolumn{2}{|c|}{ Un-standardized coefficients } & Standardized coefficients & \\
\hline Model* $^{*}$ & $\boldsymbol{B}$ & Std. error & $\boldsymbol{\beta}$ & $\boldsymbol{p}$ \\
\hline Sleep duration (hours) & -0.176 & 0.058 & -.242 & 0.0030 \\
\hline $\begin{array}{c}\text { Sleep quality } \\
\text { (assesses by PSQI score) }\end{array}$ & 0.023 & 0.015 & 0.130 & NS \\
\hline
\end{tabular}

*Adjusted for age, gender, BMI, waist circumference, waist to hip ratio, heart rate, SBP, DBP, and fasting blood glucose; PSQI, Pittsburgh sleep quality index.

Table-4: Multivariate logistic regression analysis for association of self-reported sleep duration and sleep quality (PSQI score) with serum hs-CRP risk group as dependent variable.

\begin{tabular}{|c|c|c|c|}
\hline Independent variables & OR & $\mathbf{9 5 \%}$ confidence Interval & $\boldsymbol{p}$ \\
\hline Sleep duration & & & $0.258-0.688$ \\
\hline Model-1 & 0.421 & $0.261-0.699$ & 0.0007 \\
\hline Model-2 & 0.427 & $0.263-0.718$ & 0.0011 \\
\hline Model-3 & 0.435 & & NS \\
\hline Sleep quality(assessed by PSQI score) & & $0.918-1.314$ & NS \\
\hline Model-1 & 1.099 & $0.927-1.334$ & NS \\
\hline Model-2 & 1.112 & $0.971-1.458$ & \\
\hline Model-3 & 1.190 & & \\
\hline
\end{tabular}

OR, Odd ratio; model-1,un-adjusted; model-2, adjusted for age, gender, BMI; model-3, adjusted for variables in model-2 plus waist circumference, waist to hip ratio, heart rate, systolic blood pressure, diastolic blood pressure, fasting blood glucose; NS, not significant.

\section{Discussion}

Our analysis provides evidence for a significant inverse association between self reported sleep duration and levels of serum hs-CRP among medical college students. Our findings are in line with an experimental study which first suggested an increase in inflammatory C-reactive protein after sleep deprivation [17].

However, previous studies investigating relationship between sleep duration and levels of hs-CRP in human have yielded conflicting results. While one previous study reported long sleep being a potential marker of underlying inflammatory illness [18], no significant association was observed between CRP levels and sleep duration in the Wisconsin Sleep Cohort Study [19]. Moreover, another study found significant variation in inflammatory markers with sleep duration only in women but not in men [20].

To the best of our knowledge, previous studies examining associations between sleep duration, sleep quality and inflammatory markers were done either in general population or in non-medical students group and none of these studies were from Indian population. Our study is the first to investigate association between self-reported sleep duration, sleep quality, and serum levels of hs-CRP among medical college students in Chhattisgarh, India.

In the present study, serum hs-CRP levels were found to be significantly increased in both short duration and long duration sleeper groups than 7 hours sleeper group. Our findings are supported by previous report that indicated that both acute total and short-term partial sleep deprivation resulted in elevated high-sensitivity CRP concentrations. Sleep loss might be one of the ways that inflammatory processes were activated [17]. Furthermore, a recent cross sectional analysis confirmed both short sleep and reductions in sleep were associated with average levels of inflammation over a 5-year periods [12]. In a population-based cohort, persistent, and not intermittent, insomnia was associated with increased risk for all-cause and cardiopulmonary 


\section{Original Research Article}

mortality and was associated with a steeper increase in inflammation [21]. Animal experiment suggested that prolonged sleep deprivation in rats was associated with an evolving proinflammatory state [22].Sleep restriction might induce a proinflammatory state by elevating the levels of cytokines, such as interleukin (IL)-1, IL-6, IL17, and C-reactive protein (CRP) [23-25].

Serum high-sensitivity C-reactive protein (hs-CRP), a member of the pentraxin family of proteins and a nonspecific marker of low grade inflammation [7], have been shown to independently predict cardiovascular disease [8].Taken together, our observation suggests that sleep deprivation may play a significant role in the pathophysiology of cardiovascular disease through inflammatory pathway. However, further prospective studies are needed to clarify whether sleep duration and sleep quality may play a causal role in the development of cardiovascular disease.

Potential mechanisms mediating the relationship of long sleep duration with increased levels of hs-CRP are still under investigation; however, several mechanisms might involve in these associations. Firstly, compared with normal sleepers, long sleepers may have increased sleep fragmentation and more frequent awakenings, leading to changes in inflammatory markers such as elevated levels of blood interleukin-6 and C-reactive protein levels [18].On the other hand, sleep deprivation may up regulate catecholamine synthesis, and subsequently stimulate the release of inflammatory mediators [26]. Morning levels of nuclear-factor kappa beta (a protein complex linked to inflammatory response) activation, are higher after a night of sleep loss, potentially identifying nuclear-factor kappa beta transcription pathways as the molecular mechanism by which sleep influences the production of interleukin-6 and other inflammatory cytokines[27].

In both linear and logistic regression analysis model, short sleep duration was significantly associated with elevated serum hs-CRP levels which were true even after adjustment for additional adjustment for other risk factors for cardiovascular disease. Similar observation was also made by Richardson MR et al [28].

Although, we found positive associations between poor sleep quality and elevated levels of serum hs-CRP, but associations were not significant. These observations, combined with our results suggest that association of sleep duration and sleep quality with hs-CRP is independent of other risk factor for cardiovascular disease. Positive association between poor sleep quality assessed by PSQI score and elevated hs-CRP independent of other cardiovascular risk factors, in our study, were also reported by various studies in human [29]. Several plausible biological, behavioural, and psychological pathways may lead to increased systemic inflammation, including C-reactive protein, in response to insufficient sleep.

Converging evidence suggests that sleep restriction leads to weight gain via alterations in systemic and cellular metabolism as well as behaviours associated with craving and food choices [30-32]. Release of IL-6 from adipocytes, in turn, stimulates C-reactive protein release by the liver. Sleep restriction has also been associated with increased autonomic arousal which, similarly, increases circulating C-reactive protein levels [33-36].

Our observations of both shorter and longer sleep duration than recommended 7 hours of sleep and increased prevalence of poor sleep quality among medical college students are in parallel with previous studies [37,38]. Medical students are one subgroup of the general population who appear to be especially vulnerable to poor sleep, perhaps due to the long duration and high intensity of study, clinical duties that include overnight on-call duties, work that can be emotionally challenging, and lifestyle choices [4]. Research on sleep disturbances in undergraduate medical students is of particular interest because of the known relationship between sleep and mental health and the concern that the academic demands of medical training can cause significant stress. It seems that the pathway linking sleep mechanism and risk for cardiovascular diseases is complex. Further studies to confirm and extend these observations as well as to elucidate the underlying mechanisms are clearly needed.

Limitations- The main limitations of our study are, firstly the relatively small sample size. Secondly, the present study estimated sleep duration and sleep quality only by a self-reported questionnaire in the PSQI. Although in many clinical studies, PSQI was found to be reliable and valid in the assessment of sleep problems $[39,40]$, it suffers from the same problems as other self-report inventories in that scores can be easily exaggerated or minimized by the person completing them [41]. A more objective method, for example actigraphy and polysomnography, combined with a subjective method can provide better result. Fourth, we did not consider the influence of genetic variance and environmental factors and diet, smoking and alcohol 


\section{Original Research Article}

consumption, which might play an important role in regulating hs-CRP levels. Finally, we must emphasize the cross-sectional nature of our study and therefore, no inferences of causality can be made.

\section{Conclusion}

In conclusion, our findings demonstrate that sleep duration $\geq 8$ hours or $\leq 6$ hours per day increases the serum levels of hs-CRP, thereby future risk of cardiovascular risk. Short sleep duration was significantly associated with elevated serum hs-CRP levels independent of other cardiovascular risk factors.

Sleep duration may have direct role in the pathophysiology of cardiovascular disease by a complex mechanism which is not clearly understood till date. Although, we report a high prevalence of poor sleep pattern assessed by Pittsburg sleep quality index among medical college students, no significant association of inflammatory markers with sleep quality was observed.

Long-term follow-up studies with large number of samples are needed to evaluate the impact of sleep restriction and sleep debt on chronic inflammation and to the pathogenesis and clinical course of cardiovascular disease. Sleep disturbances may serve as a marker of current or future cardiovascular problems in medical students and therefore, may serve as a screening tool to diagnose and treat students at an early stage prior to the onset of clinical or even subclinical disease.

Acknowledgments- We, authors, thank Indian Council of Medical Research (ICMR) under which this STS2018 project with reference Id: 2018-01144 was carried out. We are also grateful to Dr. R. K. Singh, Dean, Government Medical College, Rajnandgaon, C.G. for permitting and providing us all types of facilities needed to carry out this research study.

\section{Funding: Nil, Conflict of interest: None Permission of IRB: Yes}

\section{References}

1. Watson NF, Badr MS, Belenky G, et al. Recommended amount of sleep for a healthy adult: a joint consensus statement of the American academy of sleep medicine and sleep research society. Sleep. 2015 Jun 1; 38 (6):843-4. doi: 10.5665/sleep.4716.

2. Spiegel K, Leproult R, Van Cauter E. Impact of sleep debt on metabolic and endocrine function. Lancet. 1999 Oct 23;354(9188):1435-9. DOI:10.1016/S0140-6736 (99) $01376-8$.
3. Insufficient sleep is a public health problem. CDC features: data and statistics 2015. Available from: http:// www.cdc.gov/features/dssleep/.

4. Wong JG, Patil NG, Beh SL, et al. Cultivating psychological well-being in Hong Kong's future doctors. Med Teach. 2005 Dec;27(8):715-9. DOI:10. 1080/01421590500237945

5. Miller MA, Cappuccio FP. Inflammation, sleep, obesity and cardiovascular disease. Curr Vasc Pharmacol. 2007 Apr;5(2):93-102.

6. Shearer WT, Reuben JM, Mullington JM, et al. Soluble TNF-alpha receptor 1 and IL-6 plasma levels in humans subjected to the sleep deprivation model of spaceflight.JAllergyClinImmunol.2001Jan;107(1):165-70

7. Gabay C, Kushner I. Acute-phase proteins and other systemic responses to inflammation. N Engl J Med. 1999 Feb 11; 340 (6):448-54. DOI:10.1056/ NEJM 199902113400607

8.Ridker PM. High-sensitivity C-reactive protein and cardiovascular risk: rationale for screening and primary prevention.Am J Cardiol. 2003 Aug21;92(4B):17K-22K

9. Lagrand WK, Visser CA, Hermens WT, et al. Creactive protein as a cardiovascular risk factor: more than an epiphenomenon? Circulation. 1999 Jul 6;100 (1): 96-102.

10. Pasceri V, Willerson JT, Yeh ET. Direct proinflammatory effect of C-reactive protein on human endothelial cells. Circulation. 2000 Oct 31;102(18): 2165-8.

11. Chiang JK. Short duration of sleep is associated with elevated high-sensitivity C-reactive protein level in Taiwanese adults: a cross-sectional study. J Clin Sleep Med. 2014 Jul 15; 10(7):743-9. doi: 10.5664/jcsm.3862.

12. Ferrie JE, Kivimäki $M$, Akbaraly $\mathrm{TN}$, et al. Associations between change in sleep duration and inflammation: Findings on $\mathrm{C}$ - reactive protein and Interleukin 6 in the Whitehall II Study. Am J Epidemiol. 2013; 178(6): 956-61. doi: 10.1093/aje/kwt 072. Epub 2013 Jun 25.

13. Hall MH, Lee L, Matthews KA. Sleep duration during the school week is associated with C-reactive protein Risk Groups in healthy adolescents.Sleep Med. 2015; 16(1):73-78.doi:10.1016/j.sleep.2014.10.005. Epub 2014 Oct 28. 
14. Nakamura K, Sakurai M, Miura K, et al. Overall sleep status and high sensitivity $\mathrm{C}$-reactive protein: a prospective study in Japanese factory workers. J Sleep Res. 2014 Dec;23(6):717-727. doi: 10.1111/jsr.12182. Epub 2014 Aug 2.

15. Baessler A, Nadeem R, Harvey M, et al. Treatment for sleep apnea by continuous positive airway pressure improves levels of inflammatory markers - a metaanalysis. J Inflamm(Lond). 2013 Mar 22;10:13. doi: 10.1186/1476-9255-10-13. eCollection 2013.

16. Buysse DJ, Reynolds CF 3rd, Monk TH, et al. The Pittsburgh Sleep Quality Index: a new instrument for psychiatric practice and research. Psychiatry Res. 1989 May;28(2):193-213.

17. Meier-Ewert HK, Ridker PM, Rifai N, et al. Effect of sleep loss on C-reactive protein, an inflammatory marker of cardiovascular risk. J Am Coll Cardiol. 2004 Feb 18; 43(4): 678-83. DOI:10.1016/j. jacc.2003.07. 050

18. Dowd JB, Goldman N, Weinstein M. Sleep duration, sleep quality, and biomarkers of inflammation in a Taiwanese population. Ann Epidemiol. 2011 Nov; 21 (11):799-806. doi: 10.1016/j.annepidem. 2011. 07. 004. Epub 2011 Sep 1.

19. Taheri S, Austin D, Lin L, et al. Correlates of serum C-reactive protein (CRP)--no association with sleep duration or sleep disordered breathing. Sleep. 2007 Aug; 30 (8):991-6.

20. Miller MA, Kandala NB, Kivimaki M, et al. Gender differences in the cross-sectional relationships between sleep duration and markers of inflammation: Whitehall II study. Sleep. 2009 Jul;32(7):857-64.

21. Parthasarathy S, Vasquez MM, Halonen $M$ et al. Persistent insomnia is associated with mortality risk. Am J Med. 2015 Mar;128(3):268-75.e2. doi: 10.1016/j. amjmed.2014.10.015. Epub 2014 Oct 16.

22. Everson CA. Clinical assessment of blood leukocytes, serum cytokines, and serum immunoglobulins as responses to sleep deprivation in laboratory rats. Am J Physiol Regul Integr Comp Physiol. 2005 Oct; 289(4):R1054-63. Epub 2005 Jun 9.

23. Donga E, van Dijk M, van Dijk JG, et al. A single night of partial sleep deprivation induces insulin resistance in multiple metabolic pathways in healthy subjects. J Clin Endocrinol Metab. 2010 Jun;95(6): 2963-8. doi: 10.1210/jc.2009-2430. Epub 2010 Apr 6.

24. Faraut B, Nakib S, Drogou C, et al. Napping reverses the salivary interleukin- 6 and urinary norepinephrine changes induced by sleep restriction. J Clin Endocrinol Metab. 2015 Mar;100(3):E416-26. doi: 10.1210/jc.2014-2566. Epub 2015 Feb 10.

25. Van Leeuwen WM, Lehto M, Karisola $P$, et al. Sleep restriction increases the risk of developing cardiovascular diseases by augmenting proinflammatory responses through IL-17 and CRP. PLoS One. 2009; 4(2): e4589. doi: 10.1371/journal.pone.0004589. Epub 2009 Feb 25.

26. Irwin M, Thompson J, Miller C, et al. Effects of sleep and sleep deprivation on catecholamine and interleukin-2 levels in humans: clinical implications. J Clin Endocrinol Metab. 1999 Jun;84(6):1979-85.DOI: 10.1210/jcem.84.6.5788

27. Irwin MR, Wang M, Ribeiro D, et al. Sleep loss activates cellular inflammatory signaling. Biol Psychiatry. 2008 Sep 15;64(6):538-40. doi: 10.1016/j. biopsych. 2008.05.004. Epub 2008 Jun 17.

28. Richardson MR, Churilla JR. Sleep Duration and CReactive Protein in US Adults. South Med J. 2017 Apr;110(4):314-317. doi: 10.14423/SMJ.0000000 000000632.

29. Huang WY, Huang CC, Chang CC, et al. Associations of self-reported sleep quality with circulating interferon gamma-inducible protein 10 , interleukin 6, and high-sensitivity C-reactive protein in healthy menopausal women. PLoS One. 2017 Jan 6; 12(1):e0169216. doi: 10.1371/journal.pone.0169216. e Collection 2017.

30. Knutson KL. Does inadequate sleep play a role in vulnerability to obesity? Am J Hum Biol. 2012 MayJun;24(3):361-71. doi: 10.1002/ajhb.22219. Epub 2012 Jan 24.

31. Patel SR, Hu FB. Short sleep duration and weight gain: a systematic review. Obesity (Silver Spring). 2008 Mar;16(3):643-53. doi: 10.1038/oby.2007.118. Epub 2008 Jan 17.

32. Spiegel K, Leproult R, L'hermite-Balériaux M, et al. Leptin levels are dependent on sleep duration: relationships with sympathovagal balance, carbohydrate 


\section{Original Research Article}

regulation, cortisol, and thyrotropin. J Clin Endocrinol Metab. 2004 Nov; 89(11):5762-71.DOI:10.1210/ jc. 2004-1003

33. Dettoni JL, Consolim-Colombo FM, Drager LF, et al. Cardiovascular effects of partial sleep deprivation in healthy volunteers. J Appl Physiol (1985). 2012 Jul; 113 (2):232-6. doi: 10.1152/japplphysiol.01604. 2011. Epub 2012 Apr 26.

34. Sgoifo A, Buwalda B, Roos M, et al. Effects of sleep deprivation on cardiac autonomic and pituitaryadrenocortical stress reactivity in rats. Psychoneuroendocrinology. 2006 Feb;31(2):197-208. Epub 2005 Sep 12.DOI:10.1016/j.psyneuen. 2005. 06.009

35. Takase B, Akima T, Satomura K, et al. Effects of chronic sleep deprivation on autonomic activity by examining heart rate variability, plasma catecholamine, and intracellular magnesium levels. Biomed Pharmacother. 2004 Oct;58 Suppl1:S35-9.

36. Zhong $\mathrm{X}$, Hilton HJ, Gates GJ, et al. Increased sympathetic and decreased parasympathetic cardiovascular modulation in normal humans with acute sleep deprivation. J Appl Physiol (1985). 2005 Jun;98 (6): 2024-32. Epub 2005 Feb 17.
37. Azad MC, Fraser K, Rumana N,et al. Sleep Disturbances among Medical Students: A Global Perspective. J Clin Sleep Med. 2015 Jan 15;11(1): 6974. doi: $10.5664 /$ jcsm.4370.

38. Bahammam AS, Al-Khairy OK, Al-Taweel AA. Sleep habits and patterns among medical students. Neurosciences (Riyadh). 2005 Apr;10(2):159-62.

39. Spira AP, Beaudreau SA, Stone KL, et al. Reliability and validity of the Pittsburgh Sleep Quality Index and the Epworth Sleepiness Scale in older men. J Gerontol A Biol Sci Med Sci. 2012 Apr;67(4):433-9. doi: 10.1093/gerona/glr172. Epub 2011 Sep 20.

40. Backhaus J, Junghanns K, Broocks A, et al. Testretest reliability and validity of the Pittsburgh Sleep Quality Index in primary insomnia. J Psychosom Res. 2002 Sep;53(3):737-40.

41.Mollayeva T, Thurairajah $\mathrm{P}$, Burton $\mathrm{K}$, et al. The Pittsburgh sleep quality index as a screening tool for sleep dysfunction in clinical and non-clinical samples: A systematic review and meta-analysis. Sleep Med Rev. 2016 Feb;25:52-73. doi: 10.1016/j. smrv.2015.01. 009. Epub 2015 Feb 17.DOI:10.1016/j. smrv.2015. 01. 009.

\section{How to cite this article?}

Gupta P, Choudhury A.B, Pawar S.M, Gopi K. Associations between sleep duration, sleep quality and high sensitivity Creactive protein among medical students of a medical college in Chhattisgarh, India. Int J Med Res Rev 2018; 6(08): 395-402. doi:10.17511/ijmrr.2018.i08.01. 